\title{
Reliance on head versus eyes in the gaze following of great apes and human infants: the cooperative eye hypothesis
}

\author{
Michael Tomasello*, Brian Hare, Hagen Lehmann, Josep Call \\ Max Planck Institute for Evolutionary Anthropology, Deutscher Platz 6, D-04103 Leipzig, Germany
}

Received 13 January 2006; accepted 6 October 2006

\begin{abstract}
As compared with other primates, humans have especially visible eyes (e.g., white sclera). One hypothesis is that this feature of human eyes evolved to make it easier for conspecifics to follow an individual's gaze direction in close-range joint attentional and communicative interactions, which would seem to imply especially cooperative (mututalistic) conspecifics. In the current study, we tested one aspect of this cooperative eye hypothesis by comparing the gaze following behavior of great apes to that of human infants. A human experimenter "looked" to the ceiling either with his eyes only, head only (eyes closed), both head and eyes, or neither. Great apes followed gaze to the ceiling based mainly on the human's head direction (although eye direction played some role as well). In contrast, human infants relied almost exclusively on eye direction in these same situations. These results demonstrate that humans are especially reliant on eyes in gaze following situations, and thus, suggest that eyes evolved a new social function in human evolution, most likely to support cooperative (mututalistic) social interactions. (C) 2006 Elsevier Ltd. All rights reserved.
\end{abstract}

Keywords: Apes; Gaze following; Eyes; Social cognition

\section{Introduction}

One of the central puzzles of human evolution is when and how humans became so cooperative. Humans engage in frequent, large-scale, complex, even institutionalized cooperation with non-kin to a degree unprecedented among the primates, if not all animal species (Richerson and Boyd, 2005).

Humans also cooperate in some unique ways in close-range social interactions involving two or a few individuals acting together toward a concrete goal, possibly communicating as they do so. Similarly, chimpanzees hunt monkeys together in small groups (Boesch and Boesch, 1989; Mitani and Watts, 1999), and indeed, recent experimental research has established that in cooperation of this kind they (i) know when

\footnotetext{
* Corresponding author. Tel.: +49 3413550 400; fax: +49 3419952119.

E-mail addresses: tomas@eva.mpg.de (M. Tomasello), hare@eva.mpg.de (B. Hare), lehmann@eva.mpg.de (H. Lehmann), call@eva.mpg.de (J. Call).
}

they do and do not need a partner, and (ii) can distinguish helpful from unhelpful partners (Melis et al., 2006). However, there are still differences with human cooperation in terms of both cognitive and motivational bases. Warneken et al. (2006) found that young human children were much more motivated than young chimpanzees to engage in cooperation for its own sake (not just for an instrumental goal), and they communicated during the cooperation in much more complex ways than did their ape cousins.

Perhaps of special importance, humans seem especially inclined, as compared with other primates, to engage with one another in collaborative activities around objects-so-called joint attentional interactions (Bard and Vauclair, 1984; Tomasello and Carpenter, 2005). In these triadic interactions, each participant typically monitors what the other is attending to visually -including the other's monitoring of their own activities and visual attention - so as to coordinate actions more effectively (Tomasello et al., 2005). As one important example, human mothers and infants regularly engage in joint 
attentional interactions around objects, and these form the referential context within which skills with language develop (Bruner, 1983; Tomasello, 2003). Quantitatively, when human infants interact with caregivers, they engage in joint attentional interactions that average twice as long in duration as those of human-raised great apes with their caregivers, and importantly, during these interactions the duration of infants' looks to the face/eyes of the caregiver average twice as long as those of the apes (Carpenter et al., 1995). In joint attentional interactions among adult humans, as well as adult-child pairs, participants make frequent use of the visually-based pointing gesture. This gesture requires the participants to monitor the gaze direction of one another fairly closely, and pointing is not used by other primate species in their natural communication (Call and Tomasello, in press). In general, it would seem to be an advantage in initiating and maintaining collaborative/ joint attentional/communicative interactions of the human kind that one's eyes be easily visible to others in order to facilitate a shared activity-assuming that the other is a cooperative partner not overly inclined toward exploitation.

It turns out, as is well-known, that humans indeed do have especially visible eyes (Kobayashi and Kohshima, 1997). Human eyes are colored in a way that helps advertise both their presence and their gaze direction much more saliently than in other primates. Kobayashi and Kohshima (2001) examined 92 primate species (including humans) and found that 85 had exposed sclera that were uniformly brown or dark brown. Microscopic analysis showed that the brown coloration of the exposed sclera was created by pigmentation deposition in the epithelium cornea, conjunctiva, and sclera. In addition, when eye coloration was compared to facial skin coloration in a subset of 81 species (including humans), 80 species were found to have low contrast in eye and facial skin coloration (i.e., the outline of the eyes and the position of the iris were difficult to distinguish due to the similarity in color of the facial skin, sclera, and iris). The only species with a transparent conjunctiva and white sclera without any pigmentation was humans. In addition, humans were the only species in which the eye outline and the position of the iris were clearly visible, since the exposed sclera was paler than the lightest colored iris or surrounding skin. Finally, the human eye and its visible regions were found to be disproportionately large and horizontally elongated for body size (i.e., the visible regions of human eyes were bigger than that of the much larger gorilla). In a quantitative comparison, Kaplan and Rogers (2002) found that the amount of visible sclera was three times greater in humans than in orangutans (when looking straight ahead-twice as large when looking to the side).

Thus, one hypothesis is that human-type eyes evolved in the context of pressures for enhanced cooperative-communicative abilities of the kind needed in mutualistic social interactions involving joint attention and visually based communication such as pointing. At present, we know of no data directly relevant to this hypothesis. But at the same time, we know of no systematic data supporting any other hypotheses explaining the uniqueness of the human eye in terms of function (although see Kobayashi and Kohshima, 2001, for some interesting related data). One approach is to compare the way that humans and their closest primate relatives follow the gaze direction of others, specifically, in the extent to which they use the eyes versus the head in gaze following. There is relevant information in previous studies of gaze following in both nonhuman primates and human infants, but in none of these has the distinction between following the direction of the head alone versus following the direction of the eyes alone been systematically tested. It is thus unclear if humans make special use of the eyes as compared with other primate species including great apes.

In experimental paradigms with great apes in which the subject must determine whether a human can see her or not-for example, before gesturing - the results are mixed. In some studies, chimpanzees and other apes care whether the human is bodily oriented toward them (e.g., Povinelli and Eddy, 1996a; Liebal et al., 2004), but they do not seem to care whether the human's eyes are open or closed (Povinelli and Eddy, 1996a; Kaminski et al., 2004). Other studies, however, have found that some apes were sensitive to whether the human's eyes were open or closed in such situations (Call and Tomasello, 1994; Gómez, 1996), and other studies have indicated that monkeys can take into account the state of the human's eyes in competitive situations (Vick and Anderson, 2003; Flombaum and Santos, 2005) and in some gaze following situations (Deaner and Platt, 2003). In experimental paradigms in which subjects have to use human-given cues to locate a hidden reward-the so-called object choice paradigm - the evidence is again mixed. Numerous studies have shown that apes and monkeys find it difficult to use the head or eye orientation of a human experimenter to locate a hidden reward (see Call and Tomasello, 2003, for a review). Other studies, however, have found positive results both with face and eye direction in apes (Povinelli and Eddy, 1996b; Itakura and Tanaka, 1998). ${ }^{1}$ What is currently unknown is the relative strength of the head versus the eyes in eliciting gaze following behavior in any nonhuman primate species.

Human infants follow gaze direction to near targets during the first year of life (D'Entremont et al., 1997), and to more distant targets at around the first birthday (Carpenter et al., 1998). Infants seem to follow glancing with the eyes (no head movement) from around 18 months of age (Corkum and Moore, 1995). In the only study with a direct comparison of head and eyes, Brooks and Meltzoff (2002) found that 14month-old infants follow an adult's gaze direction more often when her eyes are open than when they are closed-and also more often when her eyes are unobstructed versus when they are covered with a blindfold. Even in human infants, however, there has been no direct comparison of the relative effectiveness of head versus eyes in eliciting gaze following behavior.

\footnotetext{
${ }^{1}$ Note that numerous nonprimate species can spontaneously use face direction in this task, but there is little evidence that they can also use eye direction (Hare et al., 1998; Tschudin et al., 2001; Scheumann and Call, 2004; Kaminski et al., 2005; but see McKinley and Sambrook, 2000), although some can be trained to use eye orientation as a discriminative cue (e.g., Miklosi et al., 1998; Vick and Anderson, 2000).
} 
In the current study, we systematically tested the role of the head versus the eyes in the gaze following of great apes and human infants. Based on humans' greater propensity for object-centered cooperative/communicative interactions (joint attentional interactions) and their especially visible eyes, our hypothesis was that human infants would be more influenced by the eyes than the head, whereas great apes would be more influenced by the head than the eyes. We tested this "cooperative eye hypothesis" in a simple $2 \times 2$ design with the two factors Head (turned or not) and Eyes (open or closed). This design enabled a direct comparison between the role of head and eyes in the gaze following of humans versus great apes. As an additional probe, we also presented these same subjects with a human with his back turned, who then looked up. This enabled us to differentiate the role of the head versus the face (front of the head) more specifically. Because of various practical considerations (e.g., ape housing), the methods used with the great apes and human infants were slightly different, and so the two studies are reported separately.

\section{Study 1: great apes}

\section{Methods}

Participants. Participants were 19 great apes housed at the Wolfgang Köhler Primate Research Center: 11 chimpanzees (Pan troglodytes) ranging in age from 4 to 27 years, 4 gorillas (Gorilla gorilla) ranging in age from 5 to 25 years, and 4 bonobos (Pan paniscus) ranging in age from 6 to 20 years. Three additional chimpanzees and five orangutans were tested but did not pay attention to the gaze cues sufficiently for their skills to be reliably assessed; specifically, subjects who failed to attend on all five trials in a given experimental condition were excluded (see below).

Procedures. Each participant was tested individually in a test room separated from a human experimenter (E) by either plexiglass or wire mesh. For testing, E sat about $1 \mathrm{~m}$ from a plexiglass panel and attracted the subject to a location directly opposite (using food if needed). E only began a trial when the subject was sitting close to the plexiglass panel and looking at his face (vocalizations to attract attention to the face were used when needed). Each trial consisted of $\mathrm{E}$ giving a looking cue up to the ceiling (no specific object present) for approximately 10 seconds. If the subject remained close and continued to attend, the next trial was given approximately 10 seconds later (if not, E attracted him back to the appropriate location). All trials were videotaped by a camera placed behind and slightly to the side of E and aimed at the subject's seated location.

Each subject received five trials in each of six experimental conditions (the four conditions from the $2 \times 2$ design plus the back of the head condition and its control; see below). The five trials of each condition were presented as a block (about one minute between blocks) with order of conditions counterbalanced across subjects to the maximum degree possible. The six experimental conditions were as follows:
- Head Only. E closed his eyes and looked immediately to the ceiling.

- Eyes Only. E kept his head stationary and glanced with his eyes to the ceiling.

- Both. E looked to the ceiling with head and eyes.

- Neither. E stared straight ahead at the subject.

- Back of Head. E sat with his back to the subject and looked up to the ceiling.

- Back Control. E sat with his back to the subject and stared straight ahead.

Videotapes were scored by the third author. A trial was scored as a non-trial if the subject clearly did not attend to the given cue. These trials were not used in the quantitative analyses. Given attention to the cue, a subject's response was scored as gaze following if he looked to the ceiling, specifically, if he oriented his head and eyes upward so that the underside of the chin could be seen on the videotape. Any other direction of gaze was scored as not gaze following. A randomly selected $20 \%$ of the trials were scored by an independent rater. Interobserver reliability was estimated by Cohen's Kappa at 0.83 , an "excellent value" (Bakeman and Gottman, 1986).

\section{Results}

Because subjects had a different number of scoreable trials in each condition (always at least one), proportions were used in all analyses. Mean proportions are presented in Table 1 . The main statistical analysis was a $2 \times 2$ ANOVA with the factors Head and Eyes - covering the first four conditions listed in the Methods section above. For the apes, movement of the head and eyes each induced looking independently: for head $\mathrm{F}(1$, $18)=27.68, p<0.001$; for eyes $\mathrm{F}(1,18)=6.69, p=0.019$. The interaction of these two factors was not statistically significant. Head seemed to be a more powerful factor than Eyes given that the effect size for the factor Head (Partial $\mathrm{Eta}=0.61)$ was almost 2.5 times larger than the effect size for the factor Eyes (Partial Eta = 0.27). Although there were too few individuals of each species to examine species differences statistically, an informal comparison of means suggested that all three species were influenced by the head and eyes in basically similar ways.

To examine whether the apes reliably followed gaze direction in the Back of Head condition, this condition was compared to the Back Control condition (last two conditions listed above). Apes followed E's look up to the ceiling more than a third of the time (0.38) when they saw this look from behind, whereas they never looked up when just staring at the back of E's stationary head-a reliable difference

\section{Table 1}

Mean proportion of looks to the target for apes in Study $1(n=19)$, as a function of whether E's head and/or eyes were directed at the target

\begin{tabular}{lccc}
\hline & & \multicolumn{2}{c}{ Eyes } \\
\cline { 3 - 4 } & & Yes & No \\
\hline Head & Yes & 0.53 & 0.35 \\
& No & 0.13 & 0.06 \\
\hline
\end{tabular}


$\mathrm{t}(18)=4.78, p<0.001$. Again, informally, this result seemed to hold equally for each of the three species.

\section{Discussion}

Both the direction of E's head and the status of his eyes affected whether apes in the current study looked to the ceiling, but the head was clearly the most important factor. Apes followed E's head direction up even if his eyes were closed, and they were more likely to follow his open eyes to the ceiling if his head was also directed up. Interestingly, apes also reliably followed E's head direction to the ceiling when they were viewing him from behind. This suggests that when apes are following head direction, they are clearly distinguishing back from front and following the direction to which the front, the face, orients. The overall conclusion is that the gaze following of great apes is mainly influenced by face direction, with the eyes playing some role as well.

\section{Study 2: human infants}

\section{Methods}

Participants. Participants were 20 human infants at 12 monthsof-age (within two weeks on either side) and 20 human infants at 18 months-of-age (within two weeks on either side). All children were from a middle-sized German city. There were approximately equal numbers of boys and girls at each age. Infants were recruited from a child database consisting of families who had previously volunteered for developmental research. Five additional infants (three 18-month-olds and two 12-month-olds) were tested but excluded from the study for failure to cooperate or parent interference.

Procedures. Parents brought their children to a child laboratory. After a brief warm-up period, the infant was seated on the parent's lap. The experimenter (E) sat directly across at a distance of about 1 to $2 \mathrm{~m}$. E only began a trial when the infant was looking to his face (vocalizations to attract attention to the face were used when needed). As in Study 1, each trial consisted of E giving a looking cue up to the ceiling (no specific object present) for approximately 10 seconds. If the subject remained attentive, the next trial was given approximately 10 seconds later. All trials were videotaped by a camera placed behind and slightly to the side of $\mathrm{E}$ and aimed at the infant.

Each subject received four trials in each of five experimental conditions (the four conditions from the $2 \times 2$ design plus the Back of Head condition; described in Study 1). The Back Control condition was not used with the infants because pilot testing revealed that many infants became upset when $\mathrm{E}$ turned his back and paid no attention to them (the Back of Head condition is thus compared with the Neither condition). The four trials of each condition were presented as a block (about one minute between blocks) with order of conditions counterbalanced across subjects.

Videotapes were scored by the third author. As in Study 1, a trial was scored as a non-trial if the subject clearly did not attend to the given cue; it was scored as gaze following if she looked to the ceiling; and it was scored as not gaze following if she looked in any other direction. A randomly selected $20 \%$ of the trials were scored by an independent rater, and interobserver reliability was estimated by Cohen's Kappa at 0.92 .

\section{Results}

Subjects had different numbers of scoreable trials in each condition (always at least one), therefore proportions were used in all analyses. Mean proportions are presented in Table 2. The main statistical analysis was a mixed $2 \times 2 \times 2$ ANOVA with the factors Age, Head, and Eyes. This analysis yielded a main effect for age, $\mathrm{F}(1,38)=4.67, p=0.037$, such that the 18-month-olds followed gaze more often overall. In terms of the experimental manipulation, the analysis identified Eyes as the only significant factor, $\mathrm{F}(1,38)=22.70$, $p<0.001$. The factor Head showed a trend towards significance, $\mathrm{F}(1,38)=3.18, p<0.083$. The interaction of these two factors was not statistically significant (nor were any other interactions). In contrast to the apes, for the children, eyes seemed to be a more powerful factor than head, since the effect size for the factor Eyes $($ Eta $=0.37)$ was more than five times larger than the effect size for the factor Head $($ Eta $=0.07)$.

Both age groups followed the back of the head reliably as compared with the control condition. Twelve-month-olds followed E's look up to the ceiling a bit less than one in five times (0.16) when they saw this look from behind, whereas they never looked up when $\mathrm{E}$ was staring straight ahead at them (0) - a reliable difference $[\mathrm{t}(19)=3.12, p=0.006]$. Eighteen-month-olds followed E's look up to the ceiling about half the time (0.49) when they saw this look from behind, whereas they looked up only infrequently (0.08) when E was staring straight ahead at them-again a reliable difference $[\mathrm{t}(19)=5.05, p<0.001]$.

\section{Discussion}

Like the apes, the human infants were influenced both by the direction of E's head and by the status of his eyes. In contrast to the apes, however, by far the most important factor for human infants was the eyes. In the overall analysis, the effect of Eyes was statistically reliable, whereas the effect of Head was only marginally so, with the effect size for Eyes being

Table 2

Mean proportion of looks to the target for human infants in Study 2 ( $n=20$ for both 12- and 18-month-old infants), as a function of whether E's head and/or eyes were directed at the target

\begin{tabular}{lcccc}
\hline & Age & \multicolumn{2}{c}{ Eyes } \\
\cline { 3 - 5 } & & & Yes & No \\
\hline Head & Yes & 12 & 0.20 & 0.06 \\
& & 18 & 0.33 & 0.05 \\
& & 12 & & 0.09 \\
& No & 18 & 0.24 & 0.08 \\
\hline
\end{tabular}


five times larger. As in the case of the apes, both ages of infants also readily followed E's head direction when he looked up and they were viewing from behind.

\section{General discussion}

The results of the current studies provide strong support for the cooperative eye hypothesis. Both great apes and human infants followed the gaze direction of a human reliably. In the condition in which E's head and eyes both were oriented upward, both apes and infants looked up seven to nine times more often than when E's head and eyes both were oriented downward (see Tables 1 and 2). However, in the conditions in which head and eye orientation were incongruent, the species showed very different patterns of gaze following. The apes tended to follow the head: they looked up approximately 2.5 times more often when only E's head was oriented up (eyes closed) than when only E's eyes were oriented up (head down). Human infants showed precisely the opposite pattern: they looked up approximately three times more often when only E's eyes were oriented up (head down) than when only E's head was oriented up (eyes closed). This pattern of results confirms that human infants are much more attuned to the eyes-at least in gaze following situations-than are their nearest primate relatives, the African great apes. Both apes and human infants followed the direction of the front of the head-the face-when the eyes were not available.

As a first set of findings, the current results may be limited in a number of ways. First, it should be noted that the human subjects were infants, whereas the ape subjects were mostly adults. It is possible that ape infants - as dependent individuals interacting mostly in close range with their mothers-would also be especially attuned to the eyes. However, in the current sample there were three chimpanzees three to five years of age - the youngest age at which apes follow gaze direction reliably in the vertical dimension (Tomasello et al., 2001) - and their pattern of performance was in no way exceptional. This is despite the fact that these individuals had been raised by humans, and there is some evidence that great apes raised by humans are more attuned to human eyes than are their mother-reared conspecifics (Call and Tomasello, 1994; Gómez, 1996; Itakura and Tanaka, 1998). Finally, the experimenter who provided the looking stimulus was of course human for both species, and this may have had some influence. It should be noted, however, that in a wide variety of paradigms, great apes follow human gaze quite reliably, and indeed in the current studies, apes' overall level of gaze following was actually higher than that of the human infants.

Gaze following is widespread in the animal kingdom with recent evidence coming from such widely diverse species as domestic goats (Kaminski et al., 2005), ravens (Bugnyar et al., 2004), and several primate species (e.g., Tomasello et al., 1998; Anderson and Mitchell, 1999). In terms of eye direction specifically, the only systematic evidence is for rhesus and pigtail macaques (Ferrari et al., 2000; Deaner and Platt, 2003). Most importantly, Deaner and Platt (2003) found that rhesus monkeys showed some sensitivity to eye direction alone in the absence of head direction. Although the situation was very different in that study (looker $=$ static picture of rhesus monkey), these results are generally consistent with ours in showing some sensitivity to eye direction. However, this does raise the possibility that relative sensitivity to the eyes and the head may differ across primate species, and this should be further investigated. In general, using head and/or face direction as the major cue makes sense for all animals, as head direction can be seen from a much larger distance than can eye direction, and the two are, of course, normally highly correlated. Additionally, if eye direction is hard to determine because of low eye visibility, individuals should privilege head direction because it offers a far more available indicator of anothers' attention (Kaminski et al., 2004). This may be particularly important in competitive situations in which misjudging where a dominant animal is looking may have dire consequences for a subordinate animal.

From the point of view of the looker, the fact that another individual exploits the information provided by its gaze direction may, in some cases, be detrimental (e.g., the other sees and gets the food first), and so, encouraging this behavior in others would seem to be risky. At the very least, individuals in constant competition with onlookers should not evolve morphological characteristics to help these others follow their gaze direction. The evolution of highly visible, human-like eyes would thus seem to imply cooperative groupmates who will not exploit the gaze direction of others to the extent that the looker is disadvantaged. It is possible that this logic applies across species more generally in a context dependent fashion, so that even great apes might pay more attention to eyes - and be less concerned about others following their eyes-in highly cooperative and/or mutualisitic situations such as grooming. This is a question for future research. However, the fact remains that humans, and only humans among primates, have developed a morphological feature-the highly visible eyethat makes their gaze direction easier for others to follow across all contexts.

Our cooperative eye hypothesis-as an extension of the signaling hypothesis of Kobayashi and Kohshima (2001)-is obviously not the only possibility. One can easily imagine that white sclera signal good health and therefore good mates. However, to our knowledge, there is no evidence for this hypothesis. It may also be that the unique morphology of the human eye is important not for indicating gaze direction in cooperative interactions, but for enforcing cooperative and altruistic behavior in others more generally. Thus, Burnham and Hare (in press; see also Haley and Fessler, in press) found that human adults contribute more in cooperative games when eye-like stimuli are visible. The hypothesis is that knowing others are watching is a strong deterrent to cheaters in cooperative situations, and so, the spying individual benefits from advertising his spying. It is also possible that humans have developed highly visible eyes in order to direct others' attention to external targets deceptively.

Needless to say, these hypotheses are not mutually exclusive, and highly visible eyes may serve all of these functions. We have simply established one function: for use as a cue to 
gaze direction. It is an advantage of the cooperative eye hypothesis that it fits so well with current evidence suggesting an overall difference in cooperativeness between humans and other apes. Indeed, in a recent study chimpanzees were found to be more skillful in using a human communicative cue to food location when it was given in a competitive, as opposed to a cooperative, context (Hare and Tomasello, 2004), whereas human two-year-olds showed the opposite pattern (Hermann and Tomasello, 2006). And as noted above, humans engage frequently in visually based communication about external objects, often with the species-unique pointing gesture, which requires both individuals to monitor the direction of attention of the other. Also, in communication, human eyes often signal various relevant emotional states (Baron-Cohen et al., 1997; Baron-Cohen et al., 2001; Adolphs, 2002; Ekman, 2003). For example, humans may signal cooperative intentions more or less directly using the eyes and other bodily expressions (e.g., the smile), whereas apes do not do this to the same extent, and highly visible eyes are not needed for this purpose. Interestingly in this regard, humans with autism seem to pay less attention to others' eyes and have more difficulty detecting when others make eye contact with them than do non-autistic individuals (Klin et al., 2002; Senju et al., 2003). They also seem to have trouble understanding how intentions can be inferred from information contained in the eye region of the face (Baron-Cohen et al., 1995, 1997; Ristic and Kingstone, 2005). Given that children with autism are known to have problems with a suite of cooperative and communicative skills, their lesser use of eyes might conceivably be seen as diagnostic of their cooperative/communicative skills more generally.

The current study thus supports the hypothesis that highly visible eyes and the way individuals use the eyes of others in social interaction are unique to humans, at least among the great apes. This may be because humans engage in special forms of cooperative/mutualistic interactions. If this cooperative eye hypothesis is correct, it would be especially useful to know when in evolution humans' highly visible eyes originated, as this would suggest a possible date for the origins of uniquely human forms of cooperation and communication.

\section{References}

Adolphs, R., 2002. Recognizing emotion from facial expressions: psychological and neurological mechanisms. Behav. Cogn. Neurosci. Rev. 1 (1), $21-62$.

Anderson, J.R., Mitchell, R.W., 1999. Macaques but not lemurs co-orient visually with humans. Folia Primatol. 70, 17-22.

Bakeman, R., Gottman, J., 1986. Observing Interaction: An Introduction to Sequential Analysis. Cambridge University Press, Cambridge.

Bard, K.A., Vauclair, J., 1984. The communicative context of object manipulation in ape and human adult-infant pairs. J. Hum. Evol. 13 (2), 181-190.

Baron-Cohen, S., Campbell, R., Karmiloff-Smith, A., Grant, J., Walker, J., 1995. Are children with autism blind to the mentalistic significance of the eyes? Br. J. Dev. Psychol. 13, 379-398.

Baron-Cohen, S., Wheelwright, J., Hill, Y., RastePlumb, I., 2001. The 'reading the mind in the eyes' test revised version: a study with normal adults, and adults with Asperger syndrome or high-functioning autism. J. Child Psychol. Psychiat. 42, 241-252.
Baron-Cohen, S., Wheelwright, S., Jolliffe, T., 1997. Is there a "language of the eyes"? Evidence from normal adults, and adults with autism or Asperger syndrome. Vis. Cogn. 4, 311-331.

Boesch, C., Boesch, H., 1989. Hunting behavior of wild chimpanzees in the Tai Forest National Park. Am. J. Phys. Anthropol. 78, 547-573.

Brooks, R., Meltzoff, A.N., 2002. The importance of eyes: how infants interpret adult looking behavior. Dev. Psychol. 38, 958-966.

Bruner, J., 1983. Child's Talk. Norton, New York.

Bugnyar, T., Stowe, M., Heinrich, B., 2004. Ravens, Corvus corax, follow gaze direction of humans around obstacles. Proc. R. Soc. Lond., B, Biol. Sci. 271, 1331-1336.

Burnham, T., Hare, B. Engineering human cooperation: does involuntary neural activation increase public goods contributions? Hum. Nat., in press.

Call, J., Tomasello, M., 1994. The production and comprehension of referential pointing by orangutans. J. Comp. Psychol. 108, 307-317.

Call, J., Tomasello, M., 2003. Social cognition. In: Maestripieri, D. (Ed.), Primate Psychology. Harvard University Press, Cambridge, MA, pp. 234-253.

Call, J., Tomasello, M. The Gestural Communication of Monkeys and Apes. Lawrence Erlbaum Publishers, in press.

Carpenter, M., Nagell, K., Tomasello, M., 1998. Social cognition, joint attention, and communicative competence from 9 to 15 months of age. Monogr. Soc. Res. Child Dev. 255.

Carpenter, M., Tomasello, M., Savage-Rumbaugh, S., 1995. Joint attention and imitative learning in children, chimpanzees, and enculturated chimpanzees. Soc. Dev. 4, 18-37.

Corkum, V., Moore, C., 1995. Development of joint visual attention in infants. In: Moore, C., Dunham, P.J. (Eds.), Joint Attention: Its Origins and Role in Development. Erlbaum, Hillsdale, NJ.

Deaner, R., Platt, M., 2003. Reflexive social attention in monkeys and humans. Curr. Biol. 13, 1609-1613.

D'Entremont, B., Hains, S., Muir, D., 1997. A demonstration of gaze following in 3- to 6-month-olds. Infant Behav. Dev. 20 (4), 569-572.

Ekman, P., 2003. Emotions Revealed: Recognizing Faces and Feelings to Improve Communication and Emotional Life. Henry Holt and Co., New York, NY.

Ferrari, P.F., Kohler, E., Fogassi, L., Gallese, V., 2000. The ability to follow eye gaze and its emergence during development in macaque monkeys. Proc. Natl. Acad. Sci. U.S.A. 97, 13997-14002.

Flombaum, J., Santos, L., 2005. Rhesus monkeys attribute perceptions to others. Curr. Biol. 15, 447-452.

Gómez, J.-C., 1996. Nonhuman primate theories of (nonhuman primate) minds: some issues concerning the origins of mindreading. In: Carruthers, P., Smith, P.K. (Eds.), Theories of Theories of Mind. Cambridge University Press, Cambridge, pp. 330-343.

Haley, K., Fessler, D. Nobody's watching? Subtle cues affect generosity in an anonymous economic game. Evol. Hum. Behav., in press.

Hare, B., Call, J., Tomasello, M., 1998. Communication of food location between human and dog (Canis familiaris). Evol. Comm. 2, 137-159.

Hare, B., Tomasello, M., 2004. Chimpanzees are more skillful in competitive than in cooperative cognitive tasks. Anim. Behav. 68, 571-581.

Hermann, E., Tomasello, M., 2006. Apes' and children's understanding of cooperative and competitive motives in a communicative situation. Dev. Sci. 9, 518-529.

Itakura, S., Tanaka, M., 1998. Use of experimenter-given cues during objectchoice tasks by chimpanzees (Pan troglodytes) and orangutan (Pongo pygmaeus), and human infants (Homo sapiens). J. Comp. Psychol. 112, $119-126$.

Kaminski, J., Call, J., Tomasello, M., 2004. Body orientation and face orientation: two factors controlling apes' begging behavior from humans. Anim. Cogn. 7, 216-223.

Kaminski, J., Riedel, J., Call, J., Tomasello, M., 2005. Domestic goats (Capra hircus) follow gaze direction and use some cues in an object choice task. Anim. Behav. 69, 11-18.

Kaplan, G., Rogers, L.J., 2002. Patterns of gazing in orangutans. Int. J. Primatol. 23, 501-526.

Klin, A., Jones, W., Schultz, R., Volkmar, F., Cohen, D., 2002. Visual fixation patterns during viewing of naturalistic social situations as predictors of 
social competence in individuals with autism. Arch. Gen. Psychiatry 59, 809-816.

Kobayashi, H., Kohshima, S., 1997. Unique morphology of the human eye. Nature 387, 767-768.

Kobayashi, H., Kohshima, S., 2001. Unique morphology of the human eye and its adaptive meaning: comparative studies on external morphology of the primate eye. J. Hum. Evol. 40, 419-435.

Liebal, K., Call, J., Tomasello, M., 2004. The use of gesture sequences by chimpanzees. Am. J. Primatol. 64, 377-396.

McKinley, J., Sambrook, T.D., 2000. Use of human-given cues by domestic dogs (Canis familiaris) and horses (Equus caballus). Anim. Cogn. 3, $13-22$.

Melis, A., Hare, B., Tomasello, M., 2006. Chimpanzees recruit the best collaborators. Science 31, 1297-1300.

Miklosi, A., Polgardi, R., Topal, J., Csanyi, V., 1998. Use of experimentergiven cues in dogs. Anim. Cogn. 1, 113-121.

Mitani, J.C., Watts, D.P., 1999. Demographic influences on the hunting behavior of chimpanzees. Am. J. Phys. Anthropol. 109, 439-454.

Povinelli, D., Eddy, T.J., 1996a. What young chimpanzees know about seeing. Monogr. Soc. Res. Child Dev. 61 (3).

Povinelli, D.J., Eddy, T.J., 1996b. Chimpanzees: joint visual attention. Psychol. Sci. 7, 129-135.

Richerson, P., Boyd, R., 2005. Not by Genes Alone. University of Chicago Press, Chicago.

Ristic, J., Kingstone, A., 2005. Taking control of reflexive social attention. Cognition B55-B65.
Scheumann, M., Call, J., 2004. The use of experimenter-given cues by South African fur seals (Arctocephalus pusillus). Anim. Cogn. 7, 224-230.

Senju, A., Yaguchi, K., Tojo, Y., Hasegawa, T., 2003. Eye contact does not facilitate detection in children with autism. Cognition 89, B43-B51.

Tomasello, M., 2003. Constructing a Language: A Usage-Based Theory of Language Acquisition. Harvard University Press.

Tomasello, M., Call, J., Hare, B., 1998. Five primate species follow the visual gaze of conspecifics. Anim. Behav. 55, 1063-1069.

Tomasello, M., Carpenter, M., 2005. The emergence of social cognition in three young chimpanzees. Monogr. Soc. Res. Child Dev. 70, no. 279.

Tomasello, M., Carpenter, M., Call, J., Behne, T., Moll, H., 2005. Understanding and sharing intentions: the origins of cultural cognition. Behav. Brain Sci. 28, 675-735.

Tomasello, M., Hare, B., Fogleman, T., 2001. The ontogeny of gaze following in chimpanzees and rhesus macaques. Anim. Behav. 61, 335-343.

Tschudin, A., Call, J., Dunbar, R., Harris, G., van der Eist, C., 2001. Comprehension of signs by dolphins (Tursiops truncatus). J. Comp. Psychol. 115, $100-105$.

Vick, S., Anderson, J.R., 2000. Learning and limits of use of eye gaze by capuchin monkeys (Cebus apella) in an object-choice task. J. Comp. Psychol. 114, 200-207.

Vick, S., Anderson, J.R., 2003. Use of human visual attention cues by olive baboons (Papio anubis) in a competitive task. J. Comp. Psychol. 117, 209-216.

Warneken, F., Chen, F., Tomasello, M., 2006. Cooperative activities in young children and chimpanzees. Child Dev. 77, 640-663. 